\title{
Expression, Purification, and Characterization of Ras Protein (BmRas1) from Bombyx mori
}

\author{
Yanping Quan, ${ }^{1,2}$ Guangqiang Liu,, ${ }^{1,2}$ Wei Yu, ${ }^{1,2}$ Zuoming Nie, ${ }^{1,2}$ Jian Chen,, \\ Zhengbing $L v,{ }^{1,2}$ and Yaozhou Zhang ${ }^{1,2}$ \\ ${ }^{1}$ Institute of Biochemistry, College of Life Sciences, Zhejiang Sci-Tech University, Zhejiang Province, Hangzhou 310018, China \\ ${ }^{2}$ Zhejiang Provincial Key Laboratory of Silkworm Bioreactor and Biomedicine, Zhejiang Province, Hangzhou 310018, China
}

Correspondence should be addressed to Yaozhou Zhang, yaozhou@zstu.edu.cn

Received 9 November 2011; Accepted 24 January 2012

Academic Editor: Soraya E. Gutierrez

Copyright (C) 2012 Yanping Quan et al. This is an open access article distributed under the Creative Commons Attribution License, which permits unrestricted use, distribution, and reproduction in any medium, provided the original work is properly cited.

\begin{abstract}
The Ras subfamily is the member of small G proteins superfamily involved in cellular signal transduction. Activation of Ras signaling causes cell growth, differentiation, and survival. Bombyx mori Ras-like protein (BmRas1) may belong to the Ras subfamily. It contained an H-N-K-Ras-like domain. The BmRas1 mRNA consisted of $1459 \mathrm{bp}$. The open reading frame contained $579 \mathrm{bp}$, encoding 192 amino acids. The protein had such secondary structures as $\alpha$-helices, extended strand, and random coil. BmRas1 was expressed successfully in E. coli BL21. The recombinant protein was purified with metal-chelating affinity chromatography. The GTPase activity of purified protein was determined by $\mathrm{FeSO}_{4}-\left(\mathrm{NH}_{4}\right)_{2} \mathrm{MoO}_{4}$ assay. The results showed that purified recombinant protein had intrinsic activity of GTPase. High titer polyclonal antibodies were generated by New Zealand rabbit immunized with purified protein. The gene expression features of BmRas1 at different stages and in different organs of the fifth instar larvae were analyzed by Western blot. The results showed that BmRas1 was expressed highly in three development stages including egg, pupae, and adult, but low expression in larva. BmRas1 was expressed in these tissues including head, malpighian tubule, genital gland, and silk gland. The purified recombinant protein would be utilized to further function studies of BmRas1.
\end{abstract}

\section{Introduction}

Ras genes were first identified as homologues of rodent sarcoma virus genes. In 1982, human DNA sequences homologous to the transforming oncogenes of the v-Harvey $(\mathrm{H}$ Ras) and Kirsten (K-Ras) rat sarcoma virus were identified in DNA sequences derived from a human bladder and a human lung cancer cell line, respectively. There are three mammalian Ras proteins: H-Ras, N-Ras, and K-Ras, which consisted of 188-189 amino acid (p21 proteins), encoded by three ras genes [1]. The Ras isoforms are highly homologous [2]. Ras proteins are positioned at the inner surface of the plasma membrane where they serve as binary molecular switches to transduce extracellular ligand-mediated stimuli into the cytoplasm to control signal transduction pathways that influence cell growth, differentiation, and apoptosis $[3,4]$. The Ras protein is the prototype of the Ras superfamily of small GTPases, which share a high degree of sequence similarity and a common three-dimensional structure, called the GTP-binding domain. This domain enables them to act as molecular switches cycling between two defined conformational states: an inactive guanosine-diphosphate (GDP-) bound and an active guanosine-triphosphate-(GTP-) bound state $[3,5,6]$. The guanine nucleotide exchange factors (GEFs) promote formation of the active Ras-GTP complex by inducing dissociation of bound GDP to allow association of the more abundant GTP, thus increasing the rate of intracellular exchange of GDP for GTP [5, 7-9].

Studies in Caenorhabditis elegans, Drosophila, and mammalian cells established the mode of action of Ras proteins [10-12]. Ras couples the signals of activated growth factor receptors to downstream effectors that interact with the active GTP-bound form of Ras. Ras effectors include protein kinases, lipid kinases, and GEFs, which transmit signals to cell nuclear, recruitment to the plasma membrane, and association with substrates [13-15]. Of these, the best characterized are the Raf kinases, also referred to as the mitogen-activated protein kinase (MAPK) cascade. MAPK 
modules include the ERK pathway, the SAPK/JNK pathway, and the p38 pathway $[7,16]$. MAPK pathways are wellconserved major signaling systems involved in the transduction of extracellular signals into cellular responses in a variety of organisms The MAPK cascades activate various substrates in the cytoplasma and the nucleus of the cell, including transcription factors. These downstream targets control cellular responses (e.g, apoptosis, proliferation, and differentiation) $[12,17,18]$.

The COOH-terminal regions of small GTP-binding proteins are classified into at least four groups: (1) Cys-A-A$\mathrm{X}$ (A, aliphatic acid; X, any amino acid); (2) Cys-A-A-Leu/ Phe; (3) Cys-X-Cys; (4) Cys-Cys [19, 20]. The Cys-A-A-X structure is furthermore subclassified into two groups: one has an additional Cys residue upstream of the Cys residue of the Cys-A-A-X structure and the other has a polybasic region. In the case of the Cys-A-A-X structure, H-Ras and $\mathrm{K}$-Ras are first farnesylated at the Cys residue followed by the proteolytic removal of the A-A-X portion and the carboxyl methylation of the exposed Cys residue [21-23].

Bombyx mori was studied to excavate its potential economic value and to explore the molecular mechanisms of the physiological development in lepidoptera insects as a model species. The silkworm genome has 28 chromosome pairs containing 4.8 billion base pairs. The complete genome was sequenced and analyzed, 18,510 genes were estimated [24]. In our laboratory, a cDNA library of silkworm pupae was constructed and the whole cDNA sequencing had been performed. We found a gene named Bombyx mori ras-like protein 1 (BmRas1) (GenBank accession no. NM_001043508) from the cDNA library. BmRas1 contained an H-N-K-Raslike domain. It may be involved in the regulation of cell growth. Here we described the expression, purification, and biochemical characterization of functional BmRas1 using an E. coli expression system. The purified recombinant protein BmRas1 was detected with GTPase activity. BmRas1 was expressed in tissue throughout four developmental stages. Subcellular localization showed BmRas1 was found on membrane, partly in cytoplasm. The further studies aimed to understand the role of BmRas1 in development and biological function of Bombyx mori.

\section{Materials and Methods}

2.1. Animals and Tissues. The Bombyx mori strain used in this study is the progeny of Qingsong $\times$ Baiyu. Silkworms were reared on mulberry leaves at $25^{\circ} \mathrm{C}$ and $60-90 \%$ relative humidity in natural light. Fifth instar larvae, pupae, moths, and nascent eggs were frozen in liquid nitrogen and stored at $-80^{\circ} \mathrm{C}$. Malpighian tubule, head, epidermis, fatty body, seminal glands, ovary, and silk glands were dissected from fifth instar larvae, frozen immediately in liquid nitrogen, and stored at $-80^{\circ} \mathrm{C}$.

2.2. Bioinformatics Analysis. The protein sequences of Ras homology proteins in some species were retrieved from NCBI Protein database. Amino acid sequence of BmRas1 protein was compared with those of some members of the Ras family, which included BmRas2 (AB206960),
BmRas3 (AB170011), Aedes aegypti (EAT46745, EAT38763, EAT35784), Anopheles gambiae (XP_307965), Tribolium castaneum (XP_975587), Xenopus laevis (AAA49944), Mus musculus (NP_032310, NP_056461), Homo sapiens (NP_ 004976, NP_056461, NP_036382), Drosophila melanogaster (NP_476857, NP523917, NP476699), Caenorhabditis elegans (NP_502213), Apis mellifera (XP_394288, XP_393035), and Nasonia vitripennis (XP_001608221). Alignments of BmRas1 and Ras homology protein sequences were performed using the Jotun Hein method in DNAStar.

2.3. Plasmid Construction. A cDNA encoding BmRas1 was obtained from the cDNA library of the metaphase pupae constructed by our laboratory. Based on the cDNA sequence, two primers were designed as follows: 5 -GGGAATTCATGTCTCGAGCAGGCGACAGAC- $3^{\prime}$ and 5'-CCCTCGAGTTAAAAAAGGGTGCAATC-3', including restriction enzyme sites for EcoR I and Xho I, respectively. The predicted open reading frame was amplified. The PCR was performed for denature at $95^{\circ} \mathrm{C}$ for $5 \mathrm{~min} ; 35$ cycles of $94^{\circ} \mathrm{C}$ for $30 \mathrm{~s}, 60^{\circ} \mathrm{C}$ for $30 \mathrm{~s}, 72^{\circ} \mathrm{C}$ for $1 \mathrm{~min}$, followed by a $10-\mathrm{min}$ extension at $72^{\circ} \mathrm{C}$. The $\mathrm{PCR}$ products were purified using the E.Z.N.A.Cycle Pure Kit (Omega, USA). After digestion with $E c o$ R I and Xho I, the interested fragment was ligated into the expression vector pET-28a (+) and transformed into $E$. coli TG1 competent cells. pET-BmRas1, the positive plasmid colony with the BmRas1 gene, was sequenced subsequently by ABI 3130-xl Genetic Analyzer.

2.4. Protein Expression and Purification. The recombinant expression plasmid, pET-BmRas1, was transformed into E. coli BL21 (DE3). Bacterial cultures were incubated at $37^{\circ} \mathrm{C}$ in $\mathrm{LB}$ medium containing kanamycin until an $\mathrm{OD}_{600}$ of 0.5 was reached. Recombinant protein expression was induced by the addition of IPTG to a final concentration of $0.1 \mathrm{mM}$. Following $4 \mathrm{~h}$ incubation at $37^{\circ} \mathrm{C}$, bacteria were harvested by centrifugation and frozen at $-20^{\circ} \mathrm{C}$. Bacterial pellets were resuspended with lysis buffer $(50 \mathrm{mM}$ Tris$\mathrm{HCl}, 2 \mathrm{mM}$ EDTA, $100 \mathrm{mM} \mathrm{NaCl}, \mathrm{pH}$ 8.0) and lysed by pulsed sonication. Briefly, cell suspension was sonicated with 30 short bursts of $10 \mathrm{sec}$ followed by intervals of $20 \mathrm{sec}$ for cooling, with an Ultrasonic Crasher $\Phi 2$ cell disruptor (Ningbo Scientz, China). Keep the suspension at all times on ice. The lysates were centrifuged at $14,000 \mathrm{~g}$ for $20 \mathrm{~min}$ at $4^{\circ} \mathrm{C}$. The supernatant was collected and filtered through a $0.45 \mu \mathrm{m}$ filter (Millipore, USA). The filtrate was subjected to metal chelation column chromatography using Ni-NTA His-Bind resin (Novagen) to purify the recombinant protein, as instructed by the manufacturer. The recombinant BmRas1 proteins were separated on SDS-PAGE and verified by immunoblotting with antibodies specific for the recombinant proteins.

2.5. GTPase Activity Assay. GTPase activity of purified BmRas1 was assayed by the $\mathrm{FeSO}_{4}-\left(\mathrm{NH}_{4}\right)_{2} \mathrm{MoO}_{4}$ method [25]. The purified BmRas1 protein was transferred into dialysis bag to renaturation. The refolded protein $(100 \mu \mathrm{g} / \mathrm{mL})$ was added to the reaction buffer containing $100 \mathrm{mM}$ Tris$\mathrm{HCl}$ (pH 8.0), $0.4 \mathrm{mM}$ DTT, $650 \mu \mathrm{M} \mathrm{GTP}, 10 \mathrm{mM} \mathrm{MgCl}_{2}$ to 
$10 \mu \mathrm{g} / \mathrm{mL}$. Controls were the same reaction buffer without the BmRas1 protein or GTP. The reaction mixture was incubated at $37^{\circ} \mathrm{C}$ for $0,30,40,50,60,75,90,105$, and $120 \mathrm{~min}$. $25 \mu \mathrm{L}$ aliquots of the reaction were quenched with $5 \mu \mathrm{L}$ of $20 \%$ trichloroacetic acid (TCA). Added $\mathrm{ddH}_{2} \mathrm{O}$ to the final volume of $50 \mu \mathrm{L}$, and centrifuged at $12.000 \mathrm{~g}$ for $15 \mathrm{~min}$. The supernatant was added $200 \mu \mathrm{L} \mathrm{FeSO}_{4}-\left(\mathrm{NH}_{4}\right)_{2} \mathrm{MoO}_{4}$ solution containing $0.7 \% \mathrm{FeSO}_{4}, 0.14 \%\left(\mathrm{NH}_{4}\right)_{2} \mathrm{MoO}_{4}, 5 \mathrm{mM} \mathrm{H}_{2} \mathrm{SO}_{4}$. The amounts of hydrolyzed inorganic phosphate were measured at $660 \mathrm{~nm}$ with SpectraMax Plus384 Absorbance Microplate Reader (Molecular Devices, USA).

2.6. Antibody Preparation. A male New Zealand white rabbit was immunized with $1 \mathrm{mg}$ of purified recombinant BmRas1 protein emulsified with Freund's complete adjuvant (Sigma). Three booster doses were given at intervals of 14 days with the half amount of antigen and Freund's incomplete adjuvant (Sigma). Blood was collected after 1 month of last immunization, and the serum was isolated. The polyclonal antibody was purified by Protein A chromatography (Sigma) following the manufacturer's instructions. The purified antibody was used for immunoblotting and immunofluorescence.

2.7. Subcellular Localization. $\mathrm{BmN}$ cells were cultured to $60-70 \%$ confluence on the confocal dish. Cells were rinsed twice with $1 \mathrm{~mL}$ PBS, and fixed in $3.7 \%$ formaldehyde at $25^{\circ} \mathrm{C}$ for $10 \mathrm{~min}$. After being washed three times with PBS, cells were blocked with $3 \% \mathrm{BSA}$ at $37^{\circ} \mathrm{C}$ for $2 \mathrm{~h}$, and then incubated with anti-BmRas1 $\operatorname{IgG}(1: 1000$ dilutions) at $4^{\circ} \mathrm{C}$ for $12 \mathrm{~h}$ in contrast with negative serum as negative control. After washing three times in PBS with $0.05 \%$ Tween20 , cells were incubated with Cy3-labeled goat anti-rabbit antibody (1:1000 dilutions, Promega) and DAPI $(1: 2000$ dilution, Promega) at $37^{\circ} \mathrm{C}$ for $2 \mathrm{~h}$, following three washes in PBS with $0.05 \%$ Tween-20. Stained cells were viewed by Nikon ECLIPSE TE2000-E Confocal Microscope with image analysis software EZ-C1 3.8.

2.8. Tissues Localization. The distributions of BmRas1 in different tissues in silkworms were analyzed by Western blot. Eggs, the fifth instar larvae, pupae, moths, and tissues isolated from fifth instar larvae were ground to powder in liquid nitrogen. Powders were suspended in buffer $(50 \mathrm{mM}$ Tris pH 8.0, $0.15 \mathrm{M} \mathrm{NaCl}, 5 \mathrm{mM}$ EDTA, 0.5\% NP-40, $1 \mathrm{mM}$ DTT, $5 \mathrm{~g} / \mathrm{L}$ sodium deoxycholate, $100 \mathrm{mg} / \mathrm{L}$ PMSF, $5 \mu \mathrm{g} / \mathrm{mL}$ Aprotin) and incubated for $30 \mathrm{~min}$ on ice. Homogenates were centrifuged at $12000 \mathrm{~g}$ for $15 \mathrm{~min}$ at $4^{\circ} \mathrm{C}$. Protein concentrations in all samples were equalized before SDSPAGE. Protein extracts from each tissue were separated by $12 \%$ SDS-PAGE, and electrotransferred onto PVDF membranes (Millipore). After blocking with 3\% nonfat milk in $\mathrm{PBS}$ at $4^{\circ} \mathrm{C}$ overnight, the membranes were probed with rabbit anti-BmRas1 antibody at room temperature for 2 hours. After washing with PBST, the membranes were incubated with HRP-conjugated goat anti-rabbit IgG (BioRad Laboratories). The bands were detected by staining with diaminobenzidine method.

\section{Results}

3.1. Bioinformatics Analysis. The complete mRNA of the BmRas1 was $1459 \mathrm{bp}$ with an open reading frame (ORF) of $579 \mathrm{bp}$ encoding a protein of 192 amino acids. The predicted molecular weight of the protein was $21.8 \mathrm{kD}$ and the theoretical isoelectric point was 6.33. A homology search using BLAST (http://blast.ncbi.nlm.nih.gov/) revealed that conserved domain of BmRas1-specific hits H-N-K-Ras-like subfamily. Amino acid sequences were aligned using the algorithm Jotun Hein Method in the software package DNAStar (Figure 1). Amino acid residues of Ras superfamily members which were important to GTP/ $\mathrm{Mg}^{2+}$-binding site, GEF interaction site, Switch I and Switch II region were conserved in the BmRas1. The amino acids $32-40$ and amino acids 60-76 domains were generally referred to as the switch I and switch II domains. GEF interaction site was within the switch II domain of Ras, as residues 62-69.

The carboxy-terminal sequences of the different Ras isoforms comprising 20-25 amino acids, termed "the hypervariable region" (HVR), were highly varied between the different Ras proteins. A C-terminal CAAX motif was in diversity, which undergoes posttranslational prenylation by cymosely farnesyl transferase to generate S-farnesyl cysteine thioester, followed by proteolytic cleavage of the AAX sequence and methyl esterification of the resulting C-terminal isoprenylated cysteine in the ER.

3.2. Protein Expression and Purification. The ORF for BmRas1 was ligated into the expression plasmid, pET-28a, with a $6 \times$ His tag. The fusion protein His-BmRas1 was successfully expressed in E. coli. Recombinant proteins were separated by $12 \%$ SDS-PAGE and analyzed by Coomassie Blue staining (Figure 2). Recombinant protein was purified using Ni-NTA His · Bind resin (Novagen) columns. Purified proteins were separated by SDS-PAGE and recognized by anti-BmRas1 antibody in Western blot (Figure 3).

3.3. GTPase Activity Assay. GTPase activity of purified BmRas1 was assayed by the $\mathrm{FeSO}_{4}-\left(\mathrm{NH}_{4}\right)_{2} \mathrm{MoO}_{4}$ method to detect the amount of inorganic phosphorus. Purified proteins were incubated in the reaction mixture at $37^{\circ} \mathrm{C}$ for $0,30,45,60,75,90,105$, and $120 \mathrm{~min}$. At $30 \mathrm{~min}$, inorganic phosphorus was detected. The amount of inorganic phosphorus was increased significantly with the time, reaching the peak at $75 \mathrm{~min}$ and remaining the similar high level (Figure 4). The results showed that the purified recombinant BmRas1 protein had GTP-binding activity and hydrolysis activity without accessory protein.

3.4. Subcellular Localization. Ras proteins function as signal molecule switch, anchoring in the internal leaflet of the plasma membrane, which relay signals from a number of different cell-surface receptors to the interior of the cells and induce specific cellular responses resulting in cell growth and differentiation. Immunostaining with antibody to BmRas1 showed that BmRas1 was located on membrane, partly in cytoplasm in BmN cells (Figure 5). 


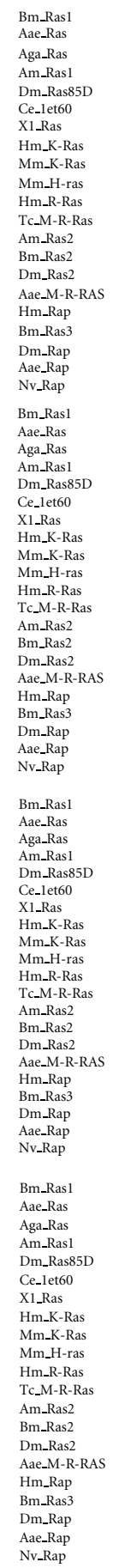

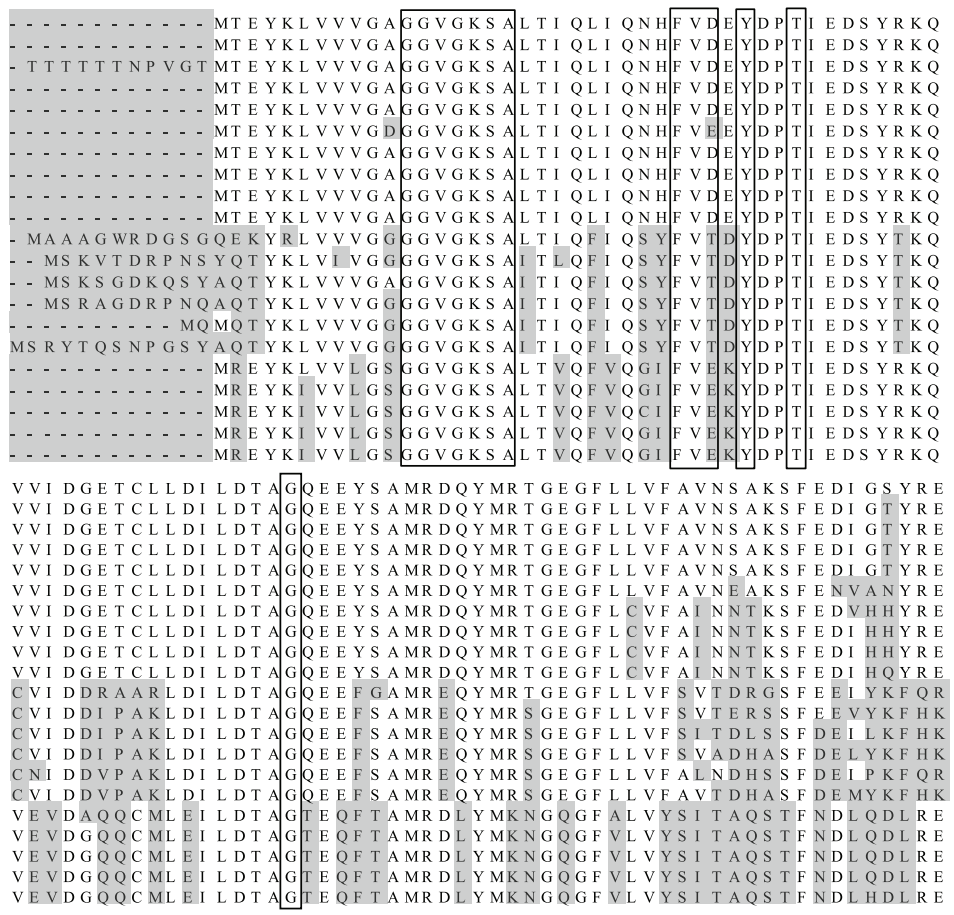

QI KRVKDAEEVPMVLVGNRCDL- QS WAVDMARAREVAQS - YNVPFVETSAKTRMC QI KRVKDAEEVPMVLVGNK D D L Q A WAVDMNQARDVAKQ-YGVPFVET SAKTRMG

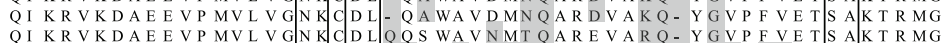

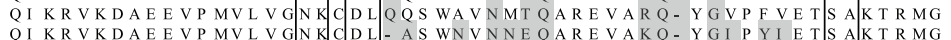

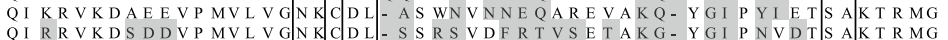

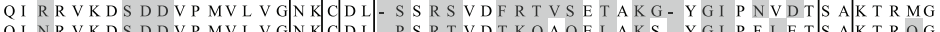

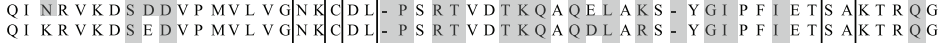

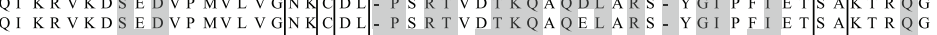
QI KRVKDSDDVP MVLVGNKADL. AARTVESRQAQDLARS - YGI PYIET SARTRQG QI LRVKDRDEFP MI LI G N K A D D D D QRQVTQEEGQQLARQ- L K VTYMEA S A I I R MN QI LRVKDRDEFP ML MVGNK DDLEHQR VVWQEEAQQLARQ- L KI P YI E C S A K MR MN QI LRVKDREE F P ML MVG N A A D D D Y H R VIEVEEAQNRARR- L KI P YI E C S A K L R MN QI L R V K DRE E F P ML I V G N K A D D E E T QR R V S L E E A Q A L S R Q- L K V P Y I E C S A K A R MN QI L R V K D R DEF P ML MVG N K C D D K K H Q Q Q VS LE E A Q N T S R N- L MI P Y I E C S A K L R V N

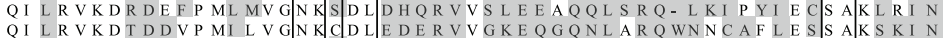
QI LRVKDTTDVPMVLVGNKTDLEAERVVGKEQGQNLARH-FNCAFMET/SAKAKI H QI LRVKDTDDVP MVLVGNKCDLEEERVVGKELGKNLAT Q- FNCAFMETSAKAKVN

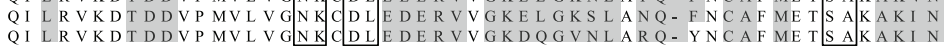

VDDAFYTLVREI - RKDKVS . . . . . . . R RKKFKGKKPRHVHKI I K Dי $\bar{C} \bar{T} \bar{L} \bar{F}$ !

VDDAFYTLVREI RKDKE . . . . . . . RGKKNRKHNKLGSSRRFK-ICRLL I

VDDAFYTLVREI - RKDKE - . . . . . . RGKKNRKHHKLVSSRRFK - 'CQLL I

VDDAFYTLVREI-RKDKEH -

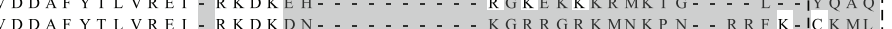

GDA Y

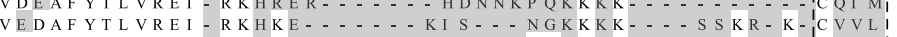

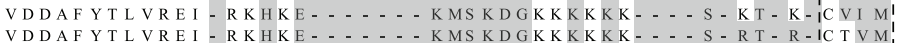

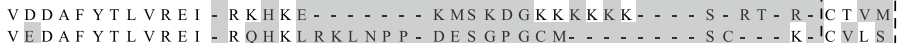

VEDAF YTLVREI - RQHKLRKLNPP - DES GP GCM. . . . .

VDQAFHELVRVI-RKFQEQ-ECPPSPEPT-RKEKDKKGCH
VDNAFYELVRVV-RKFQLS- ERPPLKTGY-MKRNKKK-

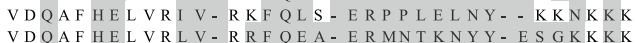

VDQAFHELVRLV-RRF QEA-ERMNTKNYY-ESGKKKK -

VDQAFHELVRIV-RKFQI A-ERPFIEQDY-KKKGKRK
VDQAFHELVRVV-RKFQLS-ERPLI DEKGKRKGGKKK

VNEI F Y DL VR QI NRKT P.

VNDVFYDLVRQI N-K-K-S

VNDI F Y DL VRQI N - K - K - S -

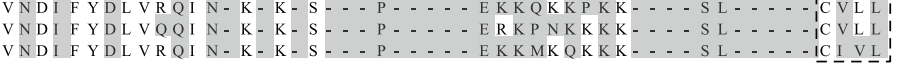

S C . . K - IC VL S I

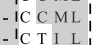

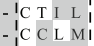

Figure 1: Alignment of amino acid sequences of Ras superfamily members. Bm, Aae, Aga, Am, Dm, Ce, Xl, Hm, Mm, Tc, and Nv mean Bombyx mori, Aedes aegypti, Anopheles gambiae, Apis mellifera, Drosophila melanogaster, Caenorhabditis elegans, Xenopus laevis, Homo sapiens, Mus musculus, Tribolium castaneum, and Nasonia vitripennis, respectively. The residues with solid light gray shade differed from BmRas1. Amino acids which are important for GTP/ $\mathrm{Mg}^{2+}$ binding were boxed in real lines. The C-terminal CAAX motif were boxed in broken lines.

3.5. Tissue Distribution. The gene expression levels of BmRas1 during different silkworm developmental stages and tissues distribution in fifth instar larvae were detected by Western blot. BmRas1 was expressed throughout four developmental stages (Figure 6). BmRas1 was expressed at high level in egg, pupae, and moth but at low level in fifth instar larva. BmRas1 was highly expressed in malpighian tubule, head, silk glands, and lowly expressed in seminal glands and ovary. No expression of BmRas1 was detected in epidermis and fatty body (Figure 7). 


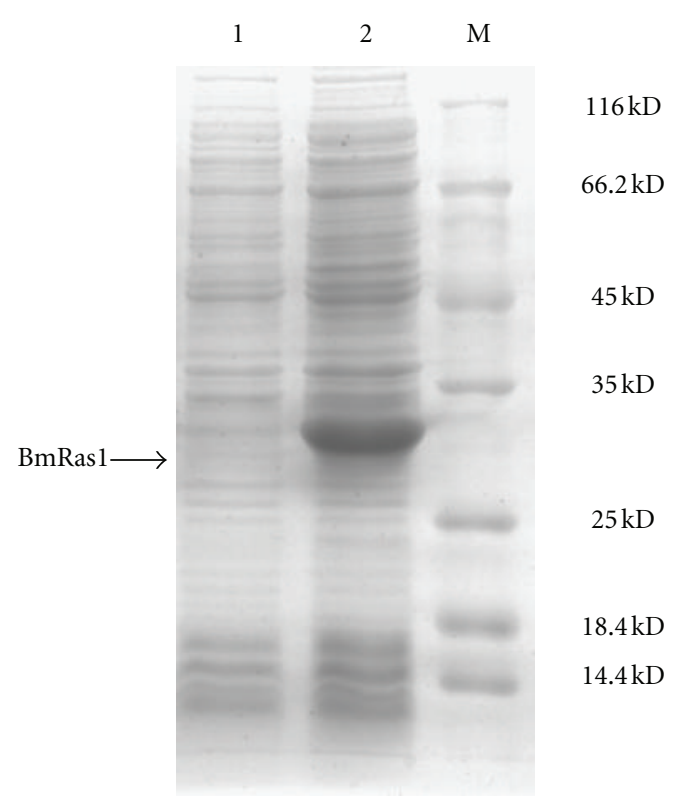

FIGURE 2: Analysis of the recombinant BmRas1 protein in SDSPAGE. Lane 1, lysates from E. coli cultures transformed with pET28 a vector plasmid after IPTG induction. Lane 2, lysates from $E$. coli cells transformed with pET-28a-BmRas1 plasmid after IPTG induction. Lane M, Protein molecular weight marker.

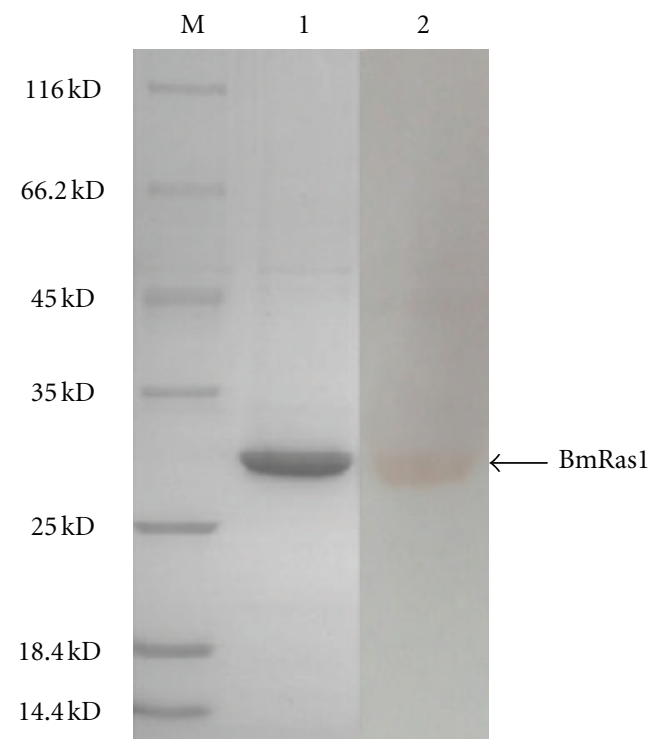

FIGURE 3: Western blot analysis of purified BmRas1 protein. Lane M. Protein molecular weight marker. Lane 1, recombinant BmRas1 was purified by nickel metal affinity resin columns and separated by SDS-PAGE. Lane 2, purified protein was analyzed by Western blot.

\section{Discussion}

Many different receptors at the cellular surface are expressed which allow cellular response to extracellular signals provided by the environment. Receptor activation by binding of ligand leads to a variety of biochemical events in which small GTPases are crucial. Ras proteins, a member of small

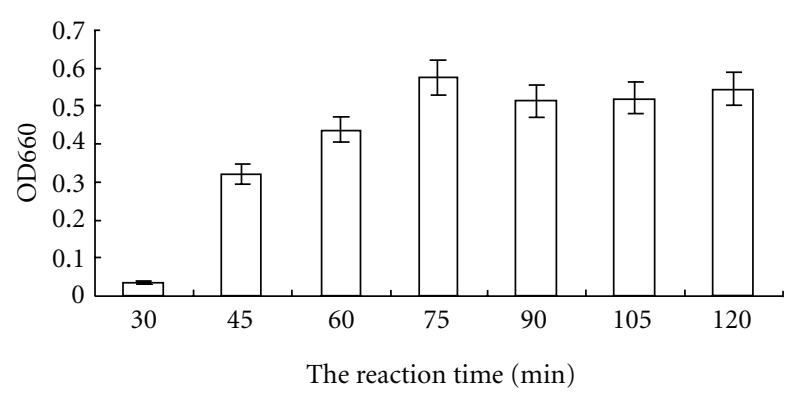

FIGURE 4: Time course of GTP hydrolysis by BmRas1. GTPase activity of purified BmRas1 was assayed by the $\mathrm{FeSO}_{4}-\left(\mathrm{NH}_{4}\right)_{2} \mathrm{MoO}_{4}$ method to detect the amount of inorganic phosphorus. Purified proteins were incubated in the reaction mixture at $37^{\circ} \mathrm{C}$ for 0,30 , $45,60,75,90,105$, and $120 \mathrm{~min}$. At $30 \mathrm{~min}$, inorganic phosphorus was detected. The amount of inorganic phosphorus was increased significantly with the time, reaching the peak at $75 \mathrm{~min}$ and remaining at the similar high level.

GTPases family, play a key role in signal transduction, proliferation, and malignant transformation. The Ras branch of the Ras superfamily presently comprises 20 proteins, that belong to various subgroups: Ras (H-Ras, K-Ras with two alternatively spliced variants expressing the A or B fourth exon, and N-Ras), Rap (with the Rap1 A and B proteins, and Rap2 A, B and C proteins), Ral (A and B), RRas (comprising the R-Ras, R-Ras2/TC-21 and R-Ras3/MRas proteins), Rit/Rin, Rheb, Di-Ras (1 and 2), and ARHI proteins [26]. The ras genes encode $21 \mathrm{kDa}$ proteins. Ras functions as a relay switch that is positioned downstream of cell surface receptor tyrosine kinases and upstream of a cytoplasmic cascade of kinases that included the mitogenactivated protein kinases (MAPKs). Activated MAPKs in turn regulated the activities of nuclear transcription factors between the cell surface and the nucleus in signaling cascade where it was defined and conserved in worms, flies, and man [2]. It is becoming increasingly evident that different members of the Ras subfamily may have different biological functions that depend not only on differences in their affinities to regulators or effectors but also in their precise subcellular localization [9]. The functions of Ras protein had been investigated in Drosophila melanogaster [27-31], but the study on the biological functions of Ras protein in Bombyx mori as representation of Lepidoptera insect was largely unknown $[32,33]$.

We conducted the research on the gene named Bombyx mori Ras protein (BmRas1) (GenBank accession no: NM_001043508) from the cDNA library. Bioinformatics analysis showed that BmRas1 contained an $\mathrm{H}-\mathrm{N}-\mathrm{K}$-Ras-like domain belonging to Ras subfamily. Amino acid sequences of some members of the Ras family from many species were aligned. BmRas1 were highly homologous with ras protein of Aedes aegypti (EAT35784), Anopheles gambiae (XP_307965), Apis mellifera (XP_394288), and Drosophila melanogaster (NP476699). The lysine-rich C-termini (last 24-25 amino acids), termed "the hypervariable region" (HVR), was highly varied, which was divided into the lipid anchor and the preceding linker domain. This domain was 
PC

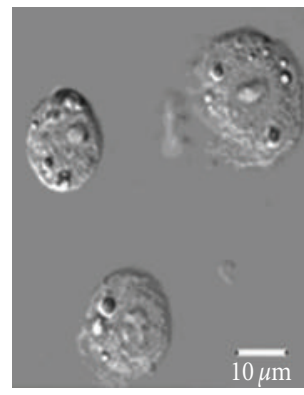

(a)

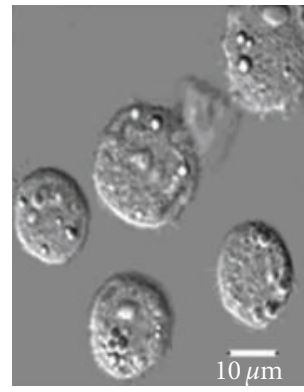

(e)

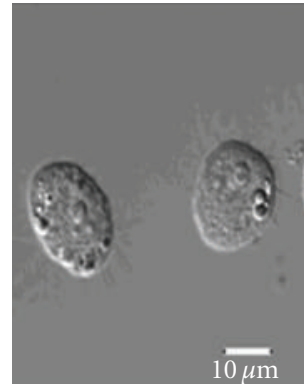

(i)

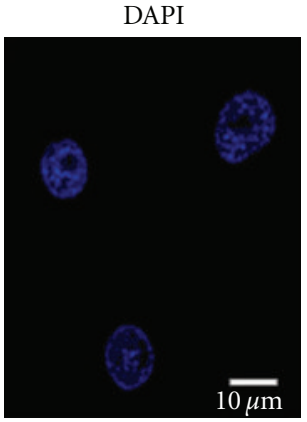

(b)

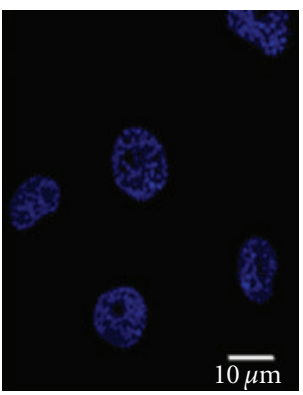

(f)

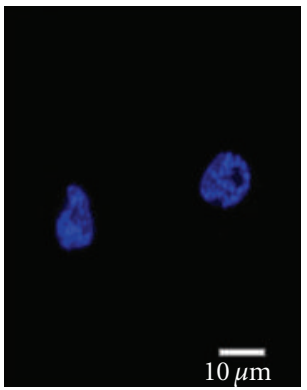

(j)

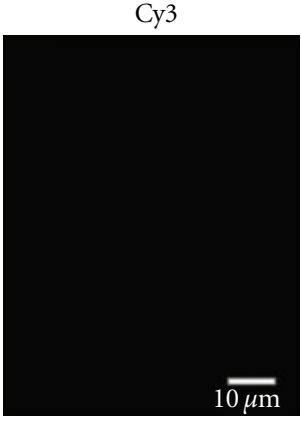

(c)

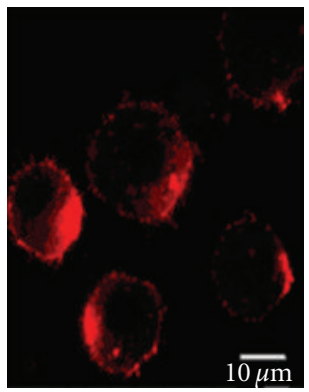

(g)

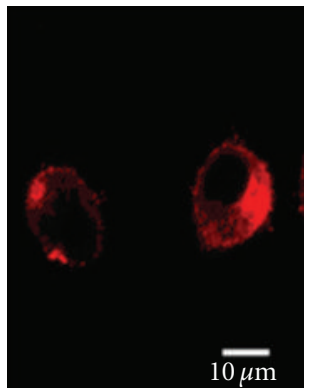

(k)

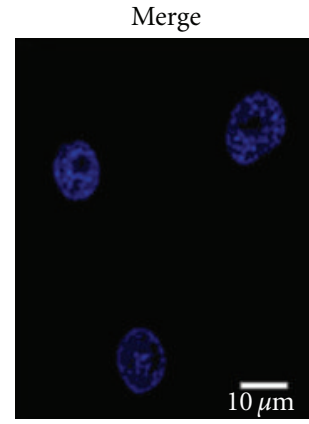

(d)

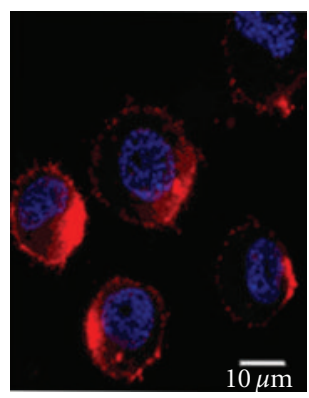

(h)

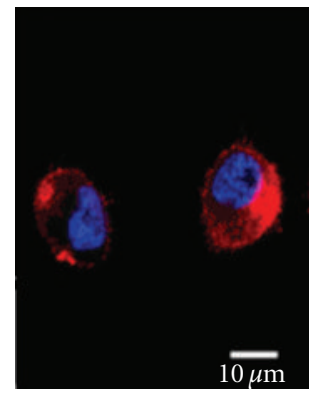

(1)

FIGURE 5: Subcellular localization of BmRas1 protein in BmN cells. Immunostaining with antibody to BmRas1 showed that BmRas1 was located on membrane, partly in cytoplasm in BmN cells. (a), (e), (i) cells in the light transmission; (b), (f), (j) nucleolus dyed by DAPI; (c), (g), (k) intracellulare BmRas1 dyed by Cy3; (d), (h), (l) merged image; (a-d) were negative control. Scar bars indicated $10 \mu \mathrm{m}$.

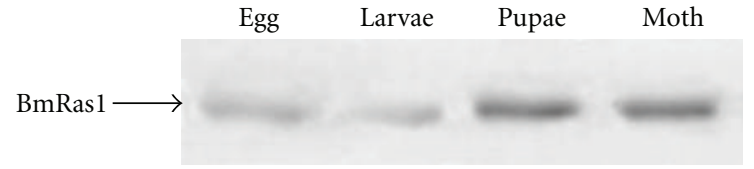

$21 \mathrm{kD}$

Figure 6: Expression of BmRas1 protein in different stages of Bombyx mori. BmRas1 expressions in four developmental stages were analyzed by Western blot. BmRas1 was expressed at high level in egg, pupae and moth, but at low level in fifth instar larva.

responsible for the membrane anchoring and intracellular trafficking of Ras protein, which interacted electrostatically with negatively charged phospholipids in the internal membrane leaflet [34]. The CAAX motif of BmRas1, CTLF, was different with all aligned sequences of other species. Bombyx mori Ras proteins were neither farnesylated nor palmitoylated but were geranylgeranylated [33].

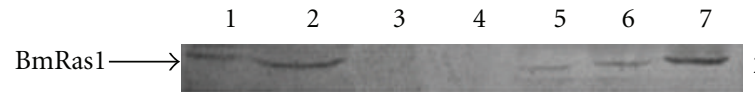

FIGURE 7: Expression of BmRas1 protein in different tissues of the fifth instar larvae of Bombyx mori was analyzed by Western blot. The BmRas1 protein was highly expressed in malpighian tubule, head, silk glands, and lowly expressed in seminal glands and ovary. No expression of BmRas1 was detected in epidermis and fatty body. 1: malpighian tubule; 2 : head; 3 : epidermis; 4 : fatty body; 5 : seminal glands; 6: ovary; 7 : silk glands.

BmRas1 gene was cloned into pET-28a $(+)$ vector and expressed in E.coli cells. Ras protein with low intrinsic GTPase activity can bind to GTP to hydrolysis. The GTPase activity of purified recombinant BmRas1 protein was tested by $\mathrm{FeSO}_{4}-\left(\mathrm{NH}_{4}\right)_{2} \mathrm{MoO}_{4}$ assay. The result showed that we 
successfully expressed the recombinant BmRas1 proteins which possessed an intrinsic GTP hydrolysis activity.

The expression of BmRas1 was detected during different developmental stages in Bombyx mori. BmRas1 protein was expressed in high level in egg, pupae, and moths, but in low expression in fifth instar larvae. In order to learn more about the distribution of BmRas1 protein, the expression levels of BmRas1 in various tissues of the fifth instar larvae were analyzed. BmRas1 proteins were expressed in tissues including malpighian tubule, head, seminal glands, ovary, and silk gland. The biological functions of BmRas1 protein in different tissues distribution need to be explored in further research. Subcellular localization of the protein can provide valuable clues about its function. The subcellular localization of the BmRas1 was examined by laser confocal microscopy. The result suggested that BmRas1 mainly localized on cellular membrane, partly in cytoplasm of $\mathrm{BmN}$ cells. The difference from Ogura's result that BmRas1 proteins were specifically localized on the cell membrane may be related to difference in cells used. Furthermore, Sf-9 cells were used in Ogura's experiment, while BmN cells were used in this research. Sf-9 Cells are derived from Spodoptera frugiperda, whereas $\mathrm{BmN}$ cells are derived from Bombyx mori. The basic research of the silkworm BmRas1 protein in tissue localization and biological functions will provide an important basis for further study of the role of this protein in silkworm.

\section{Acknowledgments}

This work was supported by financial Grants from National High Technology Research and Development Programs of China (no. 2011AA100603), National Natural Science Foundation of China (no.31101831), Zhejiang Provincial Natural Science Foundation of China (no. Y3110187, Y3090339), Zhejiang Educational Foundation (no. Y201019102), and Scientific Research Foundation of Zhejiang Sci-Tech University (no. 116151A4Y07686).

\section{References}

[1] C. J. Der, T. G. Krontiris, and G. M. Cooper, "Transforming genes of human bladder and lung carcinoma cell lines are homologous to the ras genes of Harvey and Kristen sarcoma viruses," Proceedings of the National Academy of Sciences of the United States of America, vol. 79, no. 11 I, pp. 3637-3640, 1982.

[2] S. L. Campbell, R. Khosravi-Far, K. L. Rossman, G. J. Clark, and C. J. Der, "Increasing complexity of Ras signaling," Oncogene, vol. 17, no. 11, pp. 1395-1413, 1998.

[3] R. Khosravi-Far and C. J. Der, "The Ras signal transduction pathway," Cancer and Metastasis Reviews, vol. 13, no. 1, pp. 67-89, 1994.

[4] T. Satoh and Y. Kaziro, "Ras in signal transduction," Seminars in Cancer Biology, vol. 3, no. 4, pp. 169-177, 1992.

[5] M. S. Boguski and F. McCormick, "Proteins regulating Ras and its relatives," Nature, vol. 366, no. 6456, pp. 643-654, 1993.

[6] I. R. Vetter and A. Wittinghofer, "The guanine nucleotidebinding switch in three dimensions," Science, vol. 294, no. 5545, pp. 1299-1304, 2001.
[7] E. A. Elion, "Routing MAP kinase cascades," Science, vol. 281, no. 5383, pp. 1625-1626, 1998.

[8] H. R. Bourne, D. A. Sanders, and F. McCormick, "The GTPase superfamily: conserved structure and molecular mechanism," Nature, vol. 349, no. 6305, pp. 117-127, 1991.

[9] A. Ehrhardt, G. R. A. Ehrhardt, X. Guo, and J. W. Schrader, "Ras and relatives_-job sharing and networking keep an old family together," Experimental Hematology, vol. 30, no. 10, pp. 1089-1106, 2002.

[10] A. D. Cox and C. J. Der, "Ras family signaling: therapeutic targeting," Cancer Biology \& Therapy, vol. 1, no. 6, pp. 599606, 2002.

[11] M. Wang and P. W. Sternberg, "Pattern formation during C. elegans vulval induction," Current Topics in Developmental Biology, vol. 51, pp. 189-220, 2001.

[12] J. T. Groves and J. Kuriyan, "Molecular mechanisms in signal transduction at the membrane," Nature Structural and Molecular Biology, vol. 17, no. 6, pp. 659-665, 2010.

[13] A. Rebollo and A. C. Martínez, "Ras proteins: recent advances and new functions," Blood, vol. 94, no. 9, pp. 2971-2980, 1999.

[14] A. Wittinghofer, "Signal transduction via Ras," Biological Chemistry, vol. 379, no. 8-9, pp. 933-937, 1998.

[15] M. E. Katz and F. McCormick, "Signal transduction from multiple Ras effectors," Current Opinion in Genetics and Development, vol. 7, no. 1, pp. 75-79, 1997.

[16] R. Treisman, "Regulation of transcription by MAP kinase cascades," Current Opinion in Cell Biology, vol. 8, no. 2, pp. 205215, 1996.

[17] M. J. Robinson and M. H. Cobb, "Mitogen-activated protein kinase pathways," Current Opinion in Cell Biology, vol. 9, no. 2, pp. 180-186, 1997.

[18] H. J. Schaeffer and M. J. Weber, "Mitogen-activated protein kinases: specific messages from ubiquitous messengers," Molecular and Cellular Biology, vol. 19, no. 4, pp. 2435-2444, 1999.

[19] P. J. Casey and M. C. Seabra, "Protein prenyltransferases," Journal of Biological Chemistry, vol. 271, no. 10, pp. 52895292, 1996.

[20] J. A. Glomset and C. C. Farnsworth, "Role of protein modification reactions in programming interactions between rasrelated GTPases and cell membranes," Annual Review of Cell Biology, vol. 10, pp. 181-205, 1994.

[21] J. H. Jackson, C. G. Cochrane, J. R. Bourne, P. A. Solski, J. E. Buss, and C. J. Der, "Farnesol modification of Kirsten-ras exon $4 \mathrm{~B}$ protein is essential for transformation," Proceedings of the National Academy of Sciences of the United States of America, vol. 87, no. 8, pp. 3042-3046, 1990.

[22] J. F. Hancock, A. I. Magee, J. E. Childs, and C. J. Marshall, "All ras proteins are polyisoprenylated but only some are palmitoylated," Cell, vol. 57, no. 7, pp. 1167-1177, 1989.

[23] P. J. Casey, P. A. Solski, C. J. Der, and J. E. Buss, "p21ras is modified by a farnesyl isoprenoid," Proceedings of the National Academy of Sciences of the United States of America, vol. 86, no. 21, pp. 8323-8327, 1989.

[24] Q. Xia, Z. Zhou, C. Lu et al., "A draft sequence for the genome of the domesticated silkworm (Bombyx mori)," Science, vol. 306, no. 5703, pp. 1937-1940, 2004.

[25] L. Ma, Z. Hong, and Z. Zhang, "Perinuclear and nuclear envelope localizations of Arabidopsis Ran proteins," Plant Cell Reports, vol. 26, no. 8, pp. 1373-1382, 2007.

[26] C. Der and J. Gunzburg, "Ras family proteins," in RAS Family GTPases, A. Ridley and J. Frampton, Eds., pp. 295-339, Springer, Amsterdam, The Netherlands, 2006. 
[27] H. Luo, P. E. Rose, T. M Roberts, and C. R Dearolf, “The Hopscotch Jak kinase requires the Raf pathway to promote blood cell activation and differentiation in Drosophila," Molecular Genetics and Genomics, vol. 267, no. 1, pp. 57-63, 2002.

[28] R. M. Riley, W. Jin, and G. Gibson, "Contrasting selection pressures on components of the Ras-mediated signal transduction pathway in Drosophila," Molecular Ecology, vol. 12, no. 5, pp. 1315-1323, 2003.

[29] D. A. Baker, B. Mille-Baker, S. M. Wainwright, D. IshHorowicz, and N. J. Dibb, "Mae mediates MAP kinase phosphorylation of Ets transcription factors in Drosophila," Nature, vol. 411, no. 6835, pp. 330-334, 2001.

[30] R. Gasperini and G. Gibson, "Absence of protein polymorphism in the Ras genes of Drosophila melanogaster," Journal of Molecular Evolution, vol. 49, no. 5, pp. 583-590, 1999.

[31] E. Y. Mantrova and T. Hsu, "Down-regulation of transcription factor CF2 by Drosophila Ras/MAP kinase signaling in oogenesis: cytoplasmic retention and degradation," Genes and Development, vol. 12, no. 8, pp. 1166-1175, 1998.

[32] T. Ogura, A. Tan, T. Tsubota, T. Nakakura, and T. Shiotsuki, "Identification and expression analysis of ras gene in silkworm, Bombyx mori," PLoS One, vol. 4, no. 11, article e8030, 2009.

[33] K. Moriya, T. Tsubota, N. Ishibashi et al., "Bombyx mori Ras proteins BmRas1, BmRas2 and BmRas3 are neither farnesylated nor palmitoylated but are geranylgeranylated," Insect Molecular Biology, vol. 19, no. 3, pp. 291-301, 2010.

[34] S. Eisenberg and Y. I. Henis, "Interactions of Ras proteins with the plasma membrane and their roles in signaling," Cellular Signalling, vol. 20, no. 1, pp. 31-39, 2008. 

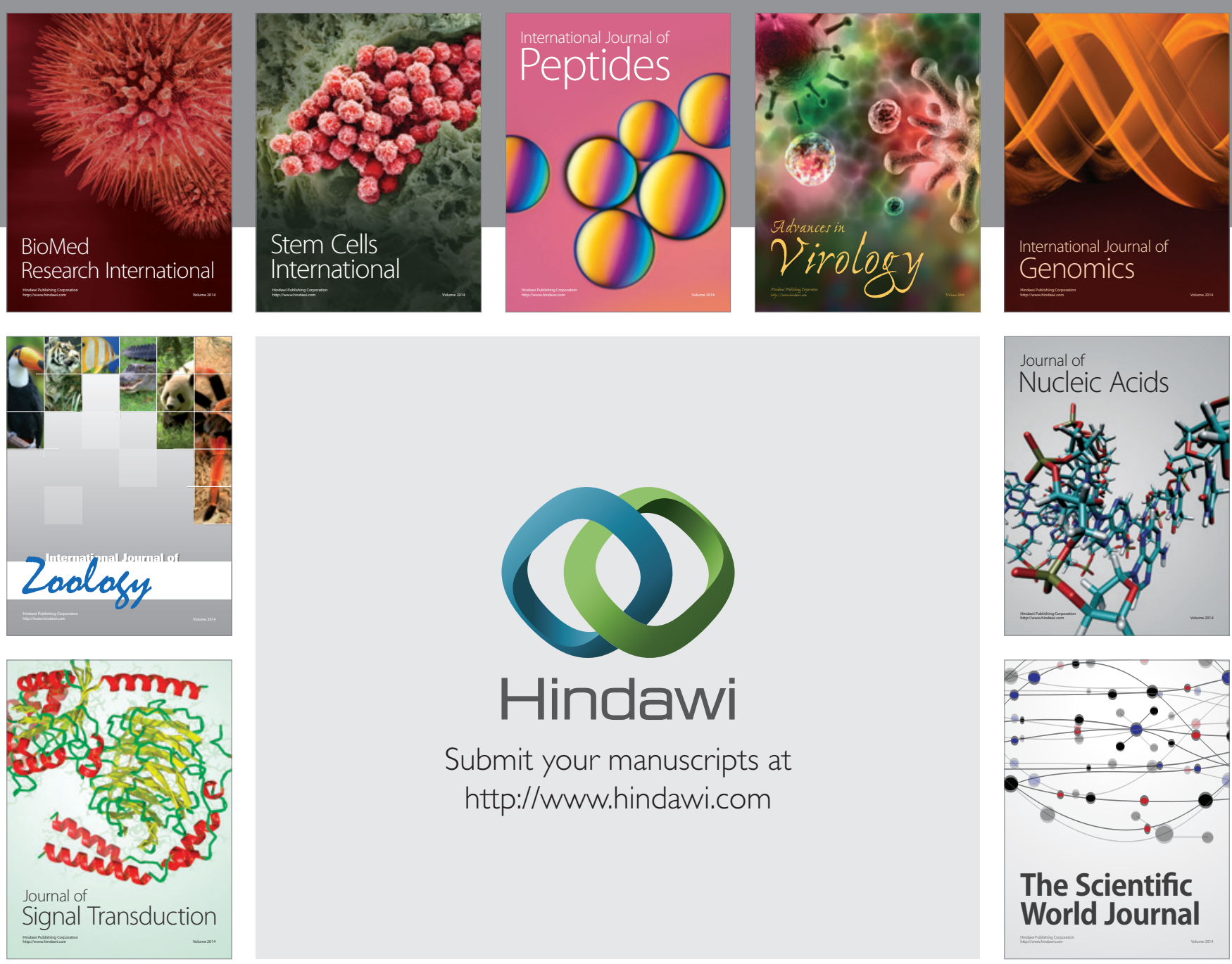

Submit your manuscripts at

http://www.hindawi.com
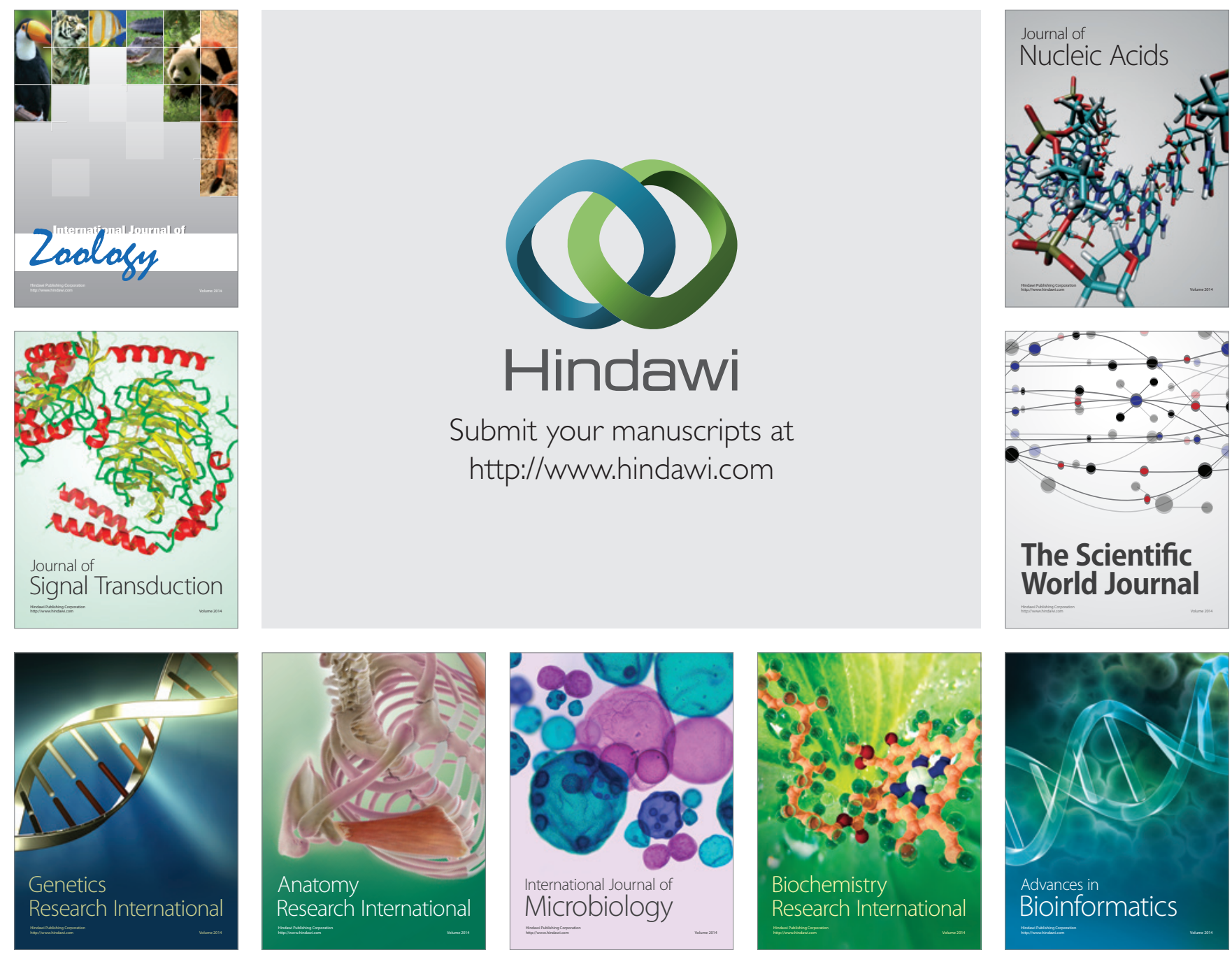

The Scientific World Journal
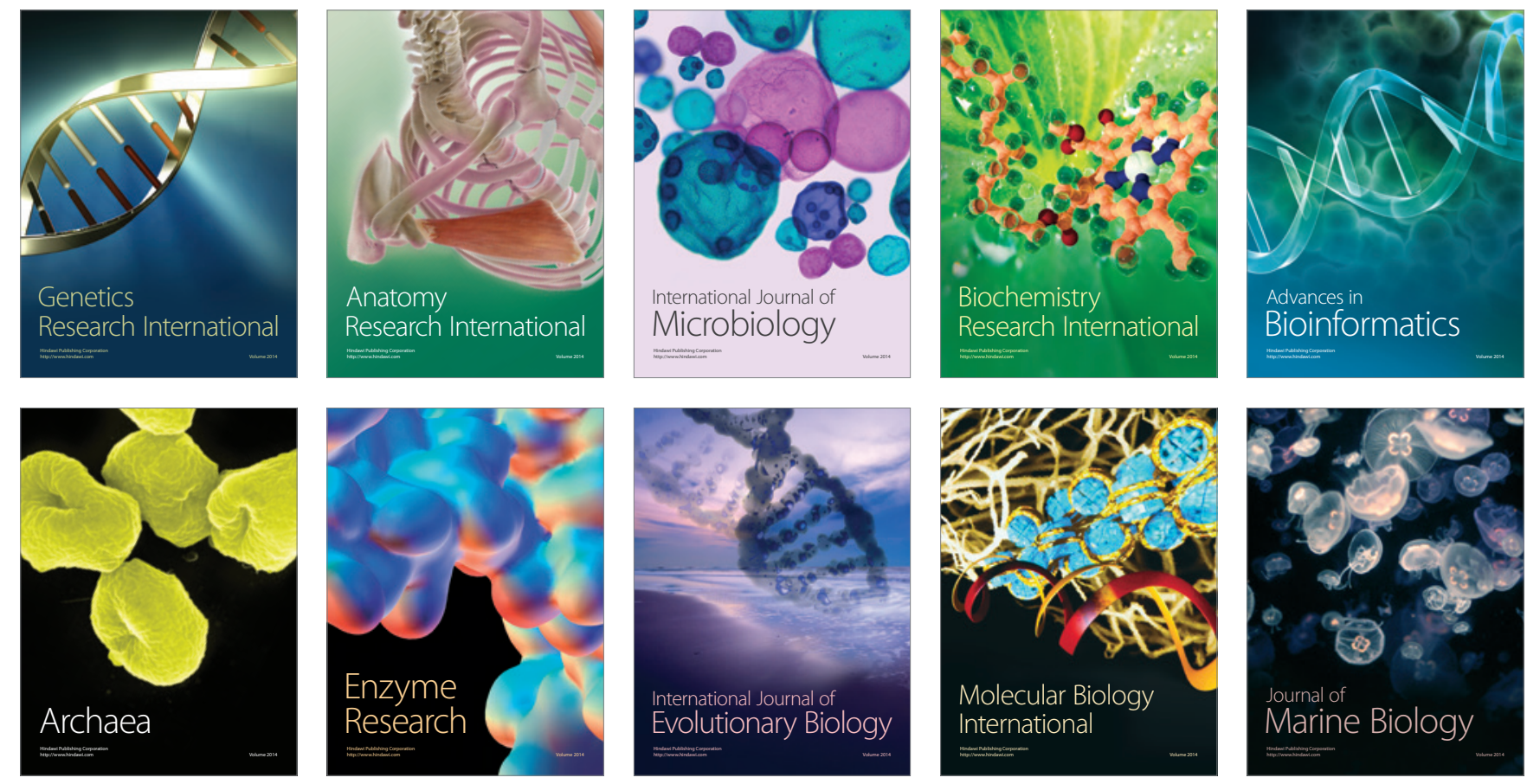